\title{
Cementum attachment protein/protein-tyrosine phosphotase-like member $A$ is not expressed in teeth
}

\author{
CHRISTOF SCHILD ${ }^{1}$, MICHAEL BEYELER ${ }^{1},{\text { NIKLAUS P. } \text { LANG }^{2} \text { and BEAT TRUEB }}^{1,3}$ \\ ${ }^{1}$ Department of Clinical Research, University of Bern, CH-3010 Bern, Switzerland; \\ ${ }^{2}$ University of Hong Kong, Faculty of Dentistry, Hong Kong SAR, P.R. China; \\ ${ }^{3}$ Department of Rheumatology, University Hospital, CH-3010 Bern, Switzerland
}

Received October 13, 2008; Accepted November 26, 2008

DOI: 10.3892/ijmm_00000130

\begin{abstract}
Cementum is a highly specialized connective tissue that covers tooth roots. The only cementum-specific protein described to date is the cementum attachment protein (CAP). A putative sequence for CAP was established from a cDNA clone isolated from a human cementifying fibroma cDNA library. This sequence overlaps with a phosphatase-like protein in muscle termed the protein-tyrosine phosphatase-like member A (PTPLA). To clarify the nature of CAP/PTPLA, we cloned the homologous rat protein and determined its sequence. The rat protein shared $94 \%$ sequence identity with the human protein. On Northern blots containing RNA from various rat tissues of different developmental stages, the cDNA hybridized to an mRNA expressed in heart and skeletal muscle but not in teeth. These results were confirmed by real-time PCR. Thus, the sequence deposited in public databanks under the name 'cementum attachment protein' does not represent genuine CAP.
\end{abstract}

\section{Introduction}

Cementum is a thin calcified layer of connective tissue that covers the root surfaces of the teeth (1). It plays a critical role during regeneration of periodontal tissues (1-3). Very little is known about this process since specific molecular markers for cementum are still lacking. The matrix of cementum resembles that of bone and consists of collagens (types I and III), fibronectin, osteopontin, bone sialoprotein, osteocalcin and vitronectin. To date, only a single cementum-specific protein, namely cementum attachment protein (CAP), has been described $(2,4)$. CAP is a collagen-like protein distinct from collagens I, XII and XIV. It promotes cell attachment of fibroblasts and other periodontal cells and its activity is mediated by $\alpha 5 \beta 1$ integrin receptors on cell surfaces. When

Correspondence to: Dr Beat Trueb, Department of Clinical Research, University of Bern, Murtenstrasse 35, PO Box 43 CH-3010 Bern, Switzerland

E-mail: beat.trueb@dkf.unibe.ch

Key words: cementum attachment protein, phosphatase-like protein, protein-tyrosine phosphotase-like member A, periodontal tissues isolated from human or bovine cementum, CAP has a molecular mass of 55-56 kDa. Two monoclonal antibodies were generated against CAP $(5,6)$. Experiments with these antibodies revealed that CAP was restricted to the cementum matrix and to cementoblasts.

Recently, a protein was cloned from a human cementifying fibroma and its sequence (GenEMBL accession number AY455942) was deposited in public databanks under the name 'cementum attachment protein'. The predicted protein consists of 140 amino acid residues with a molecular mass of $15 \mathrm{kDa}$ (AAR22554). Since this sequence is not yet described in a scientific report, its authenticity remains uncertain. The $\mathrm{N}$ terminal half of the sequence overlaps with the sequence of a novel phosphatase-like gene termed PTPLA (protein-tyrosine phosphatase-like member A) $(7,8)$. PTPLA is expressed primarily in cardiac and skeletal muscle, suggesting that it might play a role during cardiogenesis and myogenesis. Recently, mutations in the PTPLA gene were found in a dog studied as a model for autosomal recessive centronuclear myopathy (9). It is therefore concluded that impaired signaling of PTPLA might be implicated in human myopathies.

\section{Material and methods}

DNA sequencing. Expressed sequence tags (ESTs) for rat genes were obtained from the Program for Rat Gene Discovery at the University of Iowa (UI-R-AA0-wo-d-07-0-UI.s10, UIR-BJ1-atk-f-10-0-UI.s10, UIR-CN1-cji-f-16-0-UI.s10). The EST clones were sequenced by the dideoxy chain termination method with a cycle sequencing machine (ABI 3730). The sequences were analyzed with the GCG computer program (Accelrys Cambridge, UK) run on a UNIX SUN computer (Solaris 9). Databank comparisons were performed at the National Center for Biotechnology Information (www.ncbi. nlm.nih.gov).

RNA isolation and Northern blot analysis. Total RNA was extracted from various rat tissues by the guanidinium isothiocyanate method $(10,11)$ utilizing the RNeasy kit from Qiagen $\mathrm{GmbH}$ (Hilden, Germany). Samples from different developmental stages were homogenized with a polytron (Kinematica AG Lucerne, Switzerland). The purified RNA was separated on a $1 \%$ agarose gel in the presence of formaldehyde and transferred to a Nylon membrane by vacuum blotting (12). 


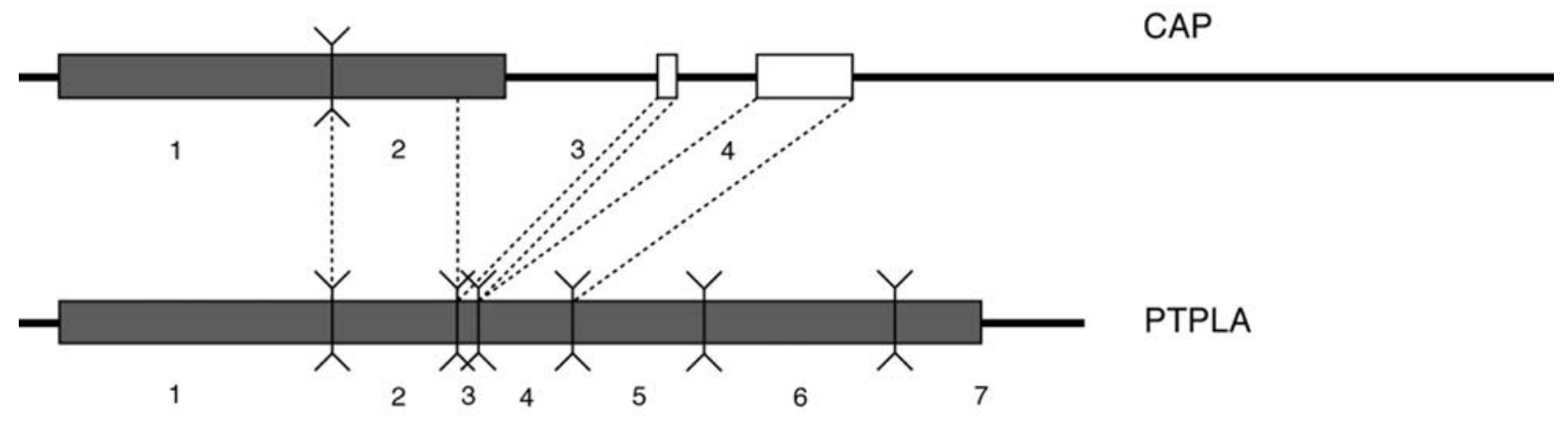

\section{Probe}

Figure 1. Schematic drawing of mRNA sequences for cementum attachment protein (CAP) (AY455942) and for the phosphatase-like protein PTPLA (AF114494). The open reading frame is shown by a grey box, the relative positions of the introns are given by brackets, and untranslated regions are indicated by a solid line. The exons are numbered below the sequence. Exons 3 and 4 that are not translated in the case of CAP are shown as open boxes. The location of the probe used for Northern blotting experiments is depicted below the sequence of PTPLA by a grey bar.

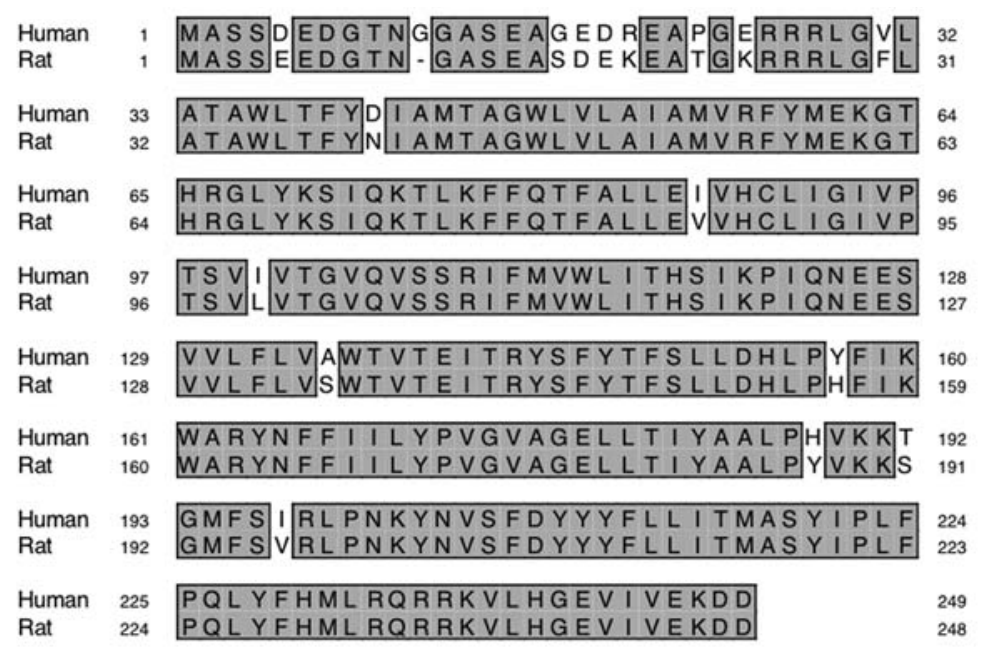

Figure 2. Alignment of the amino acid sequences for rat and human PTPLA. Identical residues are boxed. Note that the translation initiation site of the mRNAs are not yet confirmed experimentally.

The blot was hybridized overnight at $42^{\circ} \mathrm{C}$ with radioactively labeled cDNA probes in a buffer containing $50 \%$ formamide. These probes were labeled with $\left[\alpha^{-32} \mathrm{P}\right]$-dCTP by the random primed oligolabeling method $(11,13)$. After washing with standard saline citrate (SSC), the blot was exposed to X-ray film (12).

Quantitative PCR. Total RNA from various tissues was denatured at $65^{\circ} \mathrm{C}$ and cooled to room temperature. The RNA $(1 \mu \mathrm{g})$ was transcribed into first strand cDNA by reverse transcriptase from Moloney Murine Leukemia Virus (1.5 U/ $\mu \mathrm{l}$ ) as suggested by the supplier of the enzyme (Stratagene). Random hexamers served as primers. The cDNA samples were analyzed by real-time PCR with the ABI 7700 Sequence Detection System utilizing the SYBR Green PCR master mix (Applied Biosystems). The following primer pairs were used: rat S9 up GAGCTGTTGACGCTGGACGAGAAG, rat S9 low GGCCACCACCATAAGGAGAAAGGAG (annealing temperature $65^{\circ} \mathrm{C}$ ), rat CAP up CCGCCTGGCTCACCTTCT
ACAATATC, rat Cap low CCATGAAGATTCGTGAACTC ACTTGGAC (annealing temperature $65^{\circ} \mathrm{C}$ ). All experiments were performed in triplicate and normalized to results obtained with ribosomal protein $\mathrm{S} 9$.

\section{Results and discussion}

A detailed comparison of the sequences from CAP (GenEMBL accession number AY455942) and PTPLA (AF114494) suggested that the two proteins are a result of an alternative splicing event. The human PTPLA gene encompasses seven exons. The CAP sequence contains exons 1-4 but also includes introns 2, 3 and 4 (Fig. 1). Termination of translation occurs within intron 2, 16 codons downstream of the exon/intron splice boundary. When all EST clones available in the GenEMBL databank were analyzed for alternative splice variants, it was evident that PTPLA is particularly susceptible to alternative splicing. Variants missing either one or several of the exons 2, 3, 4 and 5 were identified. In addition, some 


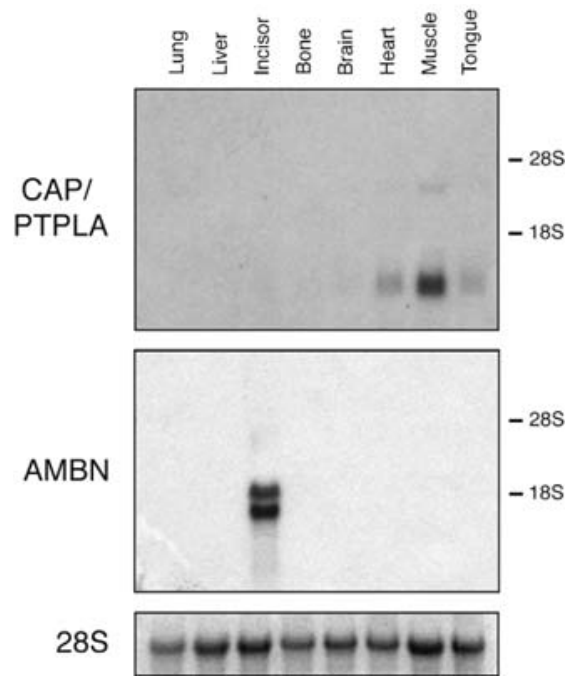

Figure 3. Expression of CAP/PTPLA in rat tissues. RNA isolated from different tissues as indicated was separated on an agarose gel, transferred to a nylon membrane and hybridized first with a probe for CAP/PTPLA, then with a probe for ameloblastin (AMBN). The migration positions of the ribosomal subunits are indicated on the right. The ribosomal subunit $28 \mathrm{~S}$ stained with ethidium bromide is included at the bottom as a loading control.

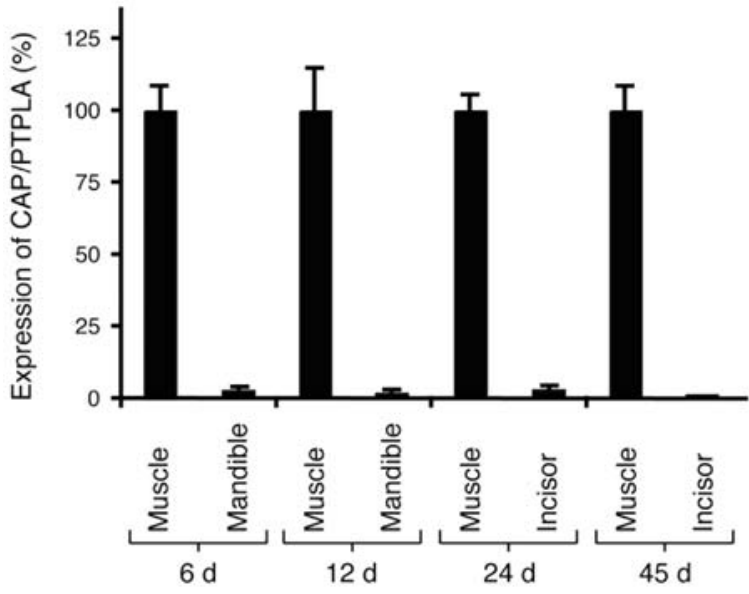

Figure 5. Quantification of the CAP/PTPLA mRNA in rat samples from different developmental stages. The RNAs were transcribed into first strand cDNAs. The expression level of CAP/PTPLA was determined by real-time PCR with a specific primer pair. The expression level was normalized to the expression level of the ribosomal protein S9 that was determined in parallel. The results are the mean of triplicates $\pm \mathrm{SE}$ and are presented in relation to the expression level in skeletal muscle (100\%). Expression of CAP/PTPLA in molars was nearly identical to that in incisors.

(14). LIM-nebulette is transcribed from an alternative promoter of the nebulette gene. It is expressed at focal adhesions of non-muscle cells whereas nebulette is found exclusively in the sarcomere of cardiac muscle cells (14).

We therefore investigated whether a similar mechanism might account for the existence of two splice variants transcribed from the CAP/PTPLA gene, one acting as the phosphatase-like protein expressed in muscles, the other acting as CAP in teeth. Clones for the homologous PTPLA protein were isolated from a rat EST clone library and sequenced completely. The assembled cDNA was found to contain 951 nucleotides with a typical polyadenylation signal and a short poly (A) tail (deposited in GenEMBL under AM260199). The rat cDNA sequence showed $88 \%$ identity with the human cDNA sequence. It harbored an open reading frame of $744 \mathrm{bp}$ that could translate into a protein of 248 amino acid residues. Compared to the human PTPLA protein, the predicted rat protein shared $94 \%$ sequence identity or $97 \%$ similarity if conservative amino acid substitutions are included (Fig. 2). A comparison with the rat genomic sequence further demonstrated that the rat gene comprised 7 exons like the human gene. The positions of all introns and the splice phases matched those of the human homologue. The PTPLA gene has therefore been highly conserved during evolution.

To analyze the tissue-specific expression of CAP/PTPLA, Northern blots were prepared with RNA extracted from 8 different rat tissues, including teeth and skeletal muscle (Fig. 3). Our cDNA probe hybridized specifically with a mRNA of $\sim 1000$ nucleotides that was expressed in skeletal muscle, heart and tongue, but not in teeth. Subsequent hybridization of the same blot with a probe specific for rat ameloblastin (positive control) yielded a typical doublet of $\sim 2000$ nucleotides that was present only in the lane for teeth (Fig. 3).

Since there is the possibility that the CAP/PTPLA gene is expressed in teeth only at a specific developmental stage, we studied recently in our laboratory concerned LIM-nebulette sequence, demonstrating that AY455942 is not simply cloning artifact.

There are several examples where two alternative transcripts from a single gene fulfill distinct functions. An example

isoforms were observed that still possessed part of the

Figure 4. Expression of CAP/PTPLA during rat development. Total RNA
from tissues obtained $6,12,24$ and 45 days after birth was processed for Northern blotting experiments. The blots were separately hybridized with The migration positions of the ribosomal subunits are indicated on the The ribosomal subunit $28 \mathrm{~S}$ stained with ethidium bromide is included at the bottom as a loading control.

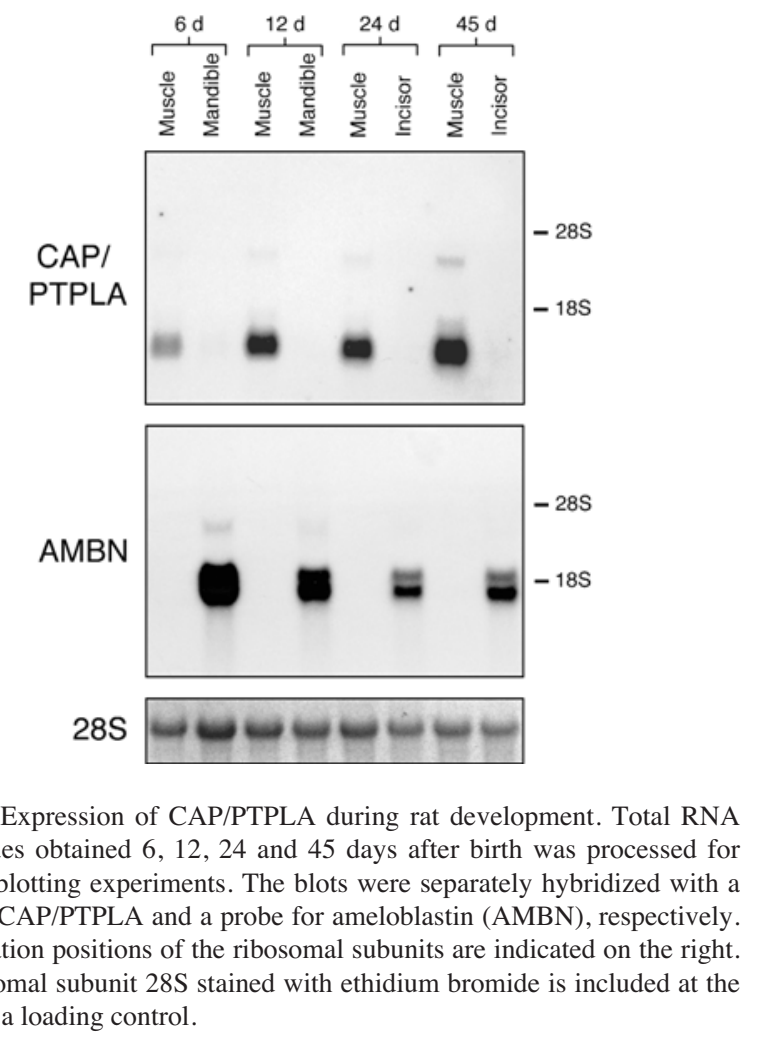


performed similar Northern blotting experiments with different tissues obtained from rats $6,12,24$ and 45 days after birth. Entire mandibles ( 6 and 12 days) as well as individual teeth (24 and 45 days) were compared with probes obtained from skeletal muscles of the same developmental stage. Incisors and molars of 45 days were analyzed in combination as well as separately. Again, expression of the CAP/PTPLA gene was restricted to muscular tissues (Fig. 4). Expression of the ameloblastin gene, however, was restricted to teeth and mandibles.

Since Northern blotting is not an extremely sensitive technique, we confirmed the results by quantitative PCR. All RNA preparations were transcribed separately into cDNA and analyzed with a primer pair specific for the CAP/PTPLA gene. The results were normalized to the expression level of the ribosomal protein $S 9$ determined in parallel in the same tissue. Expression of CAP/PTPLA was observed in all samples from the muscular tissues (Fig. 5). However, no expression of CAP/PTPLA that exceeded background noise was observed in the mandibles or in the teeth.

Our experiments clearly demonstrate that the CAP/PTPLA gene is expressed in muscles but not in teeth. The possibility that our Northern blot probe would hybridize exclusively with the normal splice variant but not with the alternatively spliced version can be ruled out since it overlaps with the entire 5' half of the alternatively spliced CAP/PTPLA mRNA. Thus, sequence AY455942 deposited in public databanks and annotated as 'cementum attachment protein' does not represent genuine CAP from teeth. This conclusion is supported by the fact that sequence AY455942 encodes a $15 \mathrm{kDa}$ protein lacking any Gly-X-Y repeats as they are typically found in collagen-like proteins. Originally, CAP was described as a collagen-like protein of $55 \mathrm{kDa}(2)$.

\section{Acknowledgements}

This study was supported by grants from the ITI Foundation (382/2005) and the Foundation for Clinical Research at the University of Bern.

\section{References}

1. Bosshardt DD: Are cementoblasts a subpopulation of osteoblasts or a unique phenotype? J Dent Res 84: 390-406, 2005.

2. Grzesik WJ and Narayanan AS: Cementum and periodontal wound healing and regeneration. Crit Rev Oral Biol Med 13: $474-484,2002$.

3. Ikezawa K, Hart CE, Williams DC and Narayanan AS: Characterization of cementum derived growth factor as an insulin-like growth factor-I like molecule. Connect Tissue Res 36: 309-319, 1997.

4. Alvarez-Perez MA, Narayanan S, Zeichner-David M, Rodriguez Carmona B and Arzate H: Molecular cloning, expression and immunolocalization of a novel human cementumderived protein (CP-23). Bone 38: 409-419, 2006.

5. Arzate H, Olson SW, Page RC, Gown AM and Narayanan AS: Production of a monoclonal antibody to an attachment protein derived from human cementum. FASEB J 6: 2990-2995, 1992.

6. Saito M, Iwase M, Maslan S, Nozaki N, Yamauchi M, Handa K, Takahashi O, Sato S, Kawase T, Teranaka T and Narayanan AS: Expression of cementum-derived attachment protein in bovine tooth germ during cementogenesis. Bone 29: 242-248, 2001.

7. Uwanogho DA, Hardcastle Z, Balogh P, Mirza G, Thornburg KL, Ragoussis J and Sharpe PT: Molecular cloning, chromosomal mapping, and developmental expression of a novel protein tyrosine phosphatase-like gene. Genomics 62: 406-416, 1999.

8. Li D, Gonzalez O, Bachinski LL and Roberts R: Human protein tyrosine phosphatase-like gene: expression profile, genomic structure, and mutation analysis in families with ARVD. Gene 256: 237-243, 2000.

9. Pele M, Tiret L, Kessler JL, Blot S and Panthier JJ: SINE exonic insertion in the PTPLA gene leads to multiple splicing defects and segregates with the autosomal recessive centronuclear myopathy in dogs. Hum Mol Genet 14: 1417-1427, 2005.

10. Chomczynski P and Sacchi N: Single-step method of RNA isolation by acid guanidinium thiocyanate-phenol-chloroform extraction. Anal Biochem 162: 156-159, 1987.

11. Trueb B, Taeschler S, Schild C and Lang NP: Expression of phosphoproteins and amelotin in teeth. Int J Mol Med 19: 49-54, 2007.

12. Ausubel FM, Brent R, Kingston RE, Moore DD, Seidman JG, Smith JA and Struhl K (eds): Current Protocols in Molecular Biology. Greene Publishing Associates, New York, 1987.

13. Feinberg AP and Vogelstein B: A technique for radiolabeling DNA restriction endonuclease fragments to high specific activity. Anal Biochem 132: 6-13, 1983.

14. Li B, Zhuang L and Trueb B: Zyxin interacts with the SH3 domains of the cytoskeletal proteins LIM-nebulette and Lasp-1. J Biol Chem 279: 20401-20410, 2004. 